\title{
Quality control for herbal medicinal plants using a sensor array (an electronic tongue)
}

\author{
Haneen Taha and Nawaf Abu-Khalaf* \\ Department of Agricultural Biotechnology, College of Agricultural Sciences and Technology, Palestine Technical \\ University - Kadoorie (PTUK), P.O.Box 7, Tulkarm, Palestine \\ *Corresponding author: n.abukhalaf@ptuk.edu.ps \\ DOI: 10.46617/icbe7001
}

\begin{abstract}
Herbal medicinal plants have a crucial role in health and social concepts worldwide. The quality of these products affects the expected remedies when using them. There are several analytical methods used for identifying the composition and following these products shelf life. The objective of this study is to use an electronic tongue (ET) for quality control of a local herbal medicinal plant product called Relax. Two multivariate data analysis (MVDA) techniques, namely principal component analysis (PCA) and hierarchical cluster analysis (HCA), were used to analyze the signals of measured samples. ET was able to follow the shelf life of the product and could identify when there was a change in its ingredients and this help in the benchmarking of the product. The obtained results may suggest the use of ET as a useful tool for herbal medicinal plant quality in the future.
\end{abstract}

Keywords: electronic tongue (ET), herbal medicinal plants, PCA, HCA, benchmarking.

\section{Introduction:}

Herbal medicinal plants play an essential role in the treatment of several diseases throughout history. There is recently an international interest in the issue of quality of these types of products. Since the quality will influence the effect of these plants on the human, the research has focused on the active ingredients and quality of herbal medicinal plants related to antimicrobial resistance and antioxidant activity effects [1].

For the antimicrobial resistance research, Silva et al. [2] tested the antimicrobial resistance against many herbal plants. The results showed potential antibiotic inhibition effect of the pomegranate (Punica granatum) fruit extract against twenty-two of the thirty-eight Staphylococcus aureus strains being tested. Moreover, a noticeable antimicrobial effect of ginger (Zingiber officinalis) oil against Staphylococcus aureus, while Escherichia coli was greatly affected by both clove (Caryophyllus aromaticus L.) and Cinnamomum zeilanicum Blume oils [2]. 
At the same time, inhibition effect was observed toward one of the tested bacteria including Staphylococcus aureus, Enterococcus faecalis, Escherichia coli, Klebsiella pneumoniae, Proteus mirabilis, Enterobacter cloacae, Morganella morganii and Pseudomonas aeruginosa. The antibacterial effect was due to at least using eleven plant species originated in Argentine [3]. The phenolic compounds presented in chamomile were found to have antimicrobial activity against Staphylococcus aureus [4].

Regarding the antioxidants activity, a group of aromatic plants in Greece were tested for their oxidation capacity, total phenolic contents, reducing power and stability through oxidation and showed consistent results [5].

Sabbobeh et al. [6] studied both antimicrobial resistance and antioxidant activity for the popular Palestinian plant Salvia palaestina (Lamiaceae) leaves. A disc diffusion method was used to estimate the antimicrobial activity of essential oils against two bacterial strains Staphylococcus aureus and Staphylococcus coli compared to gentamicin. The effect of essential oils on aureus was greater than that of gentamicin, while the essential oils and gentamicin had nearly the same activity on Escherichia coli. Moreover, there was an increase in the antioxidant activity with time and with increasing concentration of the essential oils.

Several types of equipment can be used for the quality control of herbal medicinal plants, e.g. gas chromatography (GC), gas chromatography-mass spectrometry (GC-MS), thin-layer chromatography (TLC), high-performance liquid chromatography (HPLC), capillary electrophoresis (CE), and liquid chromatography-mass (LC-M) [7, 8]. These techniques have long analytical success. On the other hand, they also have some limitations; including long analytical duration, high-cost analysis, complex sample preparations and the need to have a skilled person [9].

An electronic tongue (ET), which is a chemical sensor, can be a good alternative for analyzing the quality of herbal medicinal plants [10]. ET is composed of an array of chemical sensors with nonspecific, low selective, high stability and cross-sensitivity to different species in solution. Multivariate data analysis (MVDA) can be used for identification and quantification of analyzed samples. There are several techniques for MVDA, e.g. principal component analysis (PCA) [10,11], hierarchical cluster analysis (HCA) [12], discriminant function analysis (DFA) [13] and artificial neural network (ANN) [14].

ET has received increased attention across several domains compared to other analytical methods. Since it is fast with rapid sample preparation, reliability, useful in identifying and quantifying of several liquid compounds, acceptable accuracy and relatively low cost [15]. It has been used in many applications, e.g. biotechnology, agriculture and environment [16-28]. Moreover, ET was used for quality assessment of some taste chemicals that influence the tea [29] and herbal medicinal plants mixtures quality [30].

This work aims to investigate the possibility of using ET, as fast and reliable quality control equipment, to study the quality and stability (i.e. benchmarking) of a local herbal medical product called Relax, during one year of production. This research can open a wide window 
for using ET for further quality control applications in agricultural and biological sectors to serve the community.

\section{Materials and Methods}

\subsection{Relax herbal medicinal product}

The Relax granules product is produced by a local company called Bajjora Company, Tulkarm, Palestine. The product is used as food complement to improve gastrointestinal tract functions (i.e. herbal mixture used as a laxative), and it could influence positively in antimicrobial and antioxidant resistance. Moreover, it can be used in weight loss programs and lower cholesterol as well as harmful lipids levels. The ingredients of the product are oats, wheat germs, flax seeds, fennel, mint, caraway, anise, coriander and mahaleb cherry (Prunus mahaleb L.).

\subsection{Benchmarking of Relax granules}

Twelve different samples were collected randomly in December month (i.e. end of 2017) from Bajjora Company in Tulkarm, Palestine. Taking into consideration that each sample was produced in a different batch during the year, and there was one batch of production for this product each month (i.e. the samples covered one year of production). The manufacturing company had changed in the composition of the product, since they stopped using one of the herbs ingredients in the mixture (i.e. mahaleb cherry) from the end of February month. Table 1 shows the production date of each sample.

Table 1. Samples of Relax granules batches and their production month /2017.

\begin{tabular}{|l|l|}
\hline Sample Nr. & Production month \\
\hline 1 & January \\
\hline 2 & February* \\
\hline 3 & March \\
\hline 4 & April \\
\hline 5 & May \\
\hline 6 & June \\
\hline 7 & July \\
\hline 8 & August \\
\hline 9 & September \\
\hline 10 & October \\
\hline 11 & November \\
\hline 12 & December \\
\hline
\end{tabular}

*: The manufacturing company had stopped adding mahaleb cherry to the ingredients after the end of February. 


\subsection{Sample preparation}

Samples for herbal product testing were prepared according to previous studies [31]. For each sample, nine grams (i.e. one teaspoonful) of the product were weighted and mixed with 200 $\mathrm{ml}$ of boiled deionized water, infused for about 10 minutes and filtered by white gauze pads. Then the resulted solution was cooled to $25^{\circ} \mathrm{C}$ (room temperature) before being submitted to ET analysis.

\subsection{Electronic tongue $(E T)$}

Potentiometric electronic tongue produced by Astree II (Alpha MOS, Toulouse, France) was used. The device consists of four parts including liquid auto-sampler with 16 bakers, a sensory array, a data acquisition electronic unit and advanced software for analysis (Figure 1).

Sensors array is composed of seven solid potential sensors that are chemically modified fieldeffect transistors (ChemFET) and $\mathrm{Ag} / \mathrm{AgCl}$ reference electrode. These sensors consist of two parts: the sensitive layer and the transducer, and are coated with a specific membrane (chemical compounds) to induce both cross-sensitivity and cross-selectivity. Product samples were measured by ET according to the procedure from Alpha MOS. The average of three measurements of each sample was considered for the data analysis [32], which was carried out using PCA and HACA. Both methods are unsupervised classification methods and were used successfully in many applications [33].

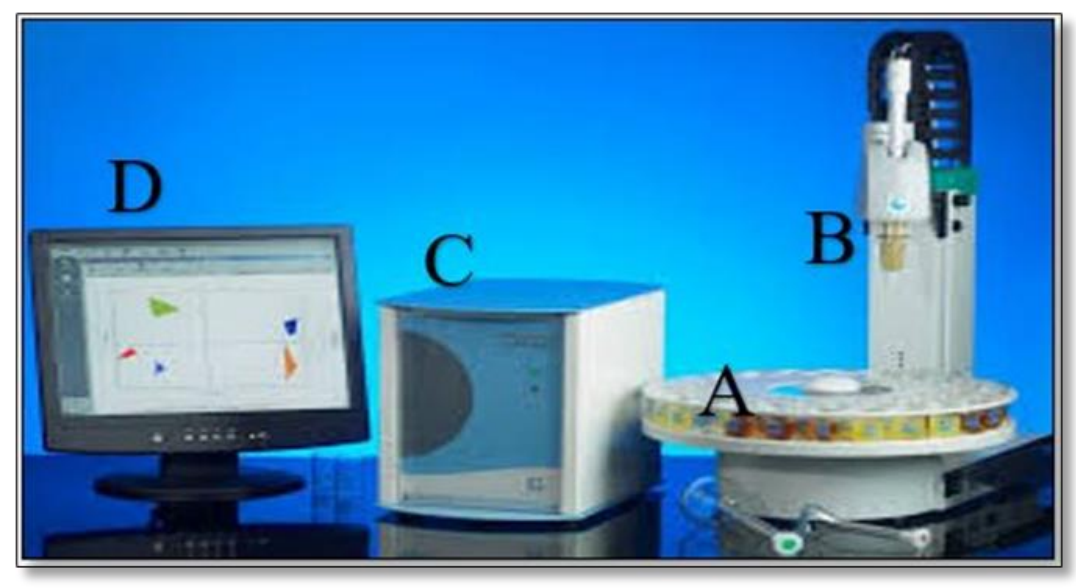

Figure 1. Alpha MOS Astree II parts: (A) liquid autosampler with a capacity for 16 bakers, (B) electrochemical sensor array, (C) acquisition unit, (D) Astree Software V12.4 (Alpha MOS, 2009).

\section{Results and discussion}

Two sensors of ET, namely ZZ and ET, were the most sensitive to Relax batches. The ET signals were stable during measurement samples. The sensors showed good reproducibility, i.e. values of standard deviation (SD) (5.6-10.4 mv) were less than $30 \mathrm{mv}$. Moreover, low 
values of the coefficient of variation (CV) (i.e. $0.31 \%-0.61 \%$ ) represented good reliability according to the manufacture of ET [32].

A PCA was carried out. Figure 2 shows the scores plot of PCA. The scores plot explains the relation between samples. Two principal components (PCs) explain 100\% of the variation of the data, which indicates that the model can explain the whole variation of the original data, without any significant loss in the information. It can be noticed that there are two main groups in the scores plot. One of the groups is for samples which were taken in January until February. The other group is for samples that were produced in March until December. The classification is most likely due to the difference in the herbal ingredients, since the company had decided to stop adding mahaleb cherry to the mixture ingredients from the end of February month. The ET could identify the difference in the herbal medicinal constituents, even though they usually added mahaleb cherry in a low quantity. This indicates that ET can detect that small difference reflecting the high sensitivity of this taste sensor in predicting the composition of samples.

It can also be noticed that all samples are relatively close to each other, indicating that the storage time (shelf-time) have little or no effect on the analyzed batches ensuring the good quality of this local product. Consequently, the products were kept in a suitable storage condition.

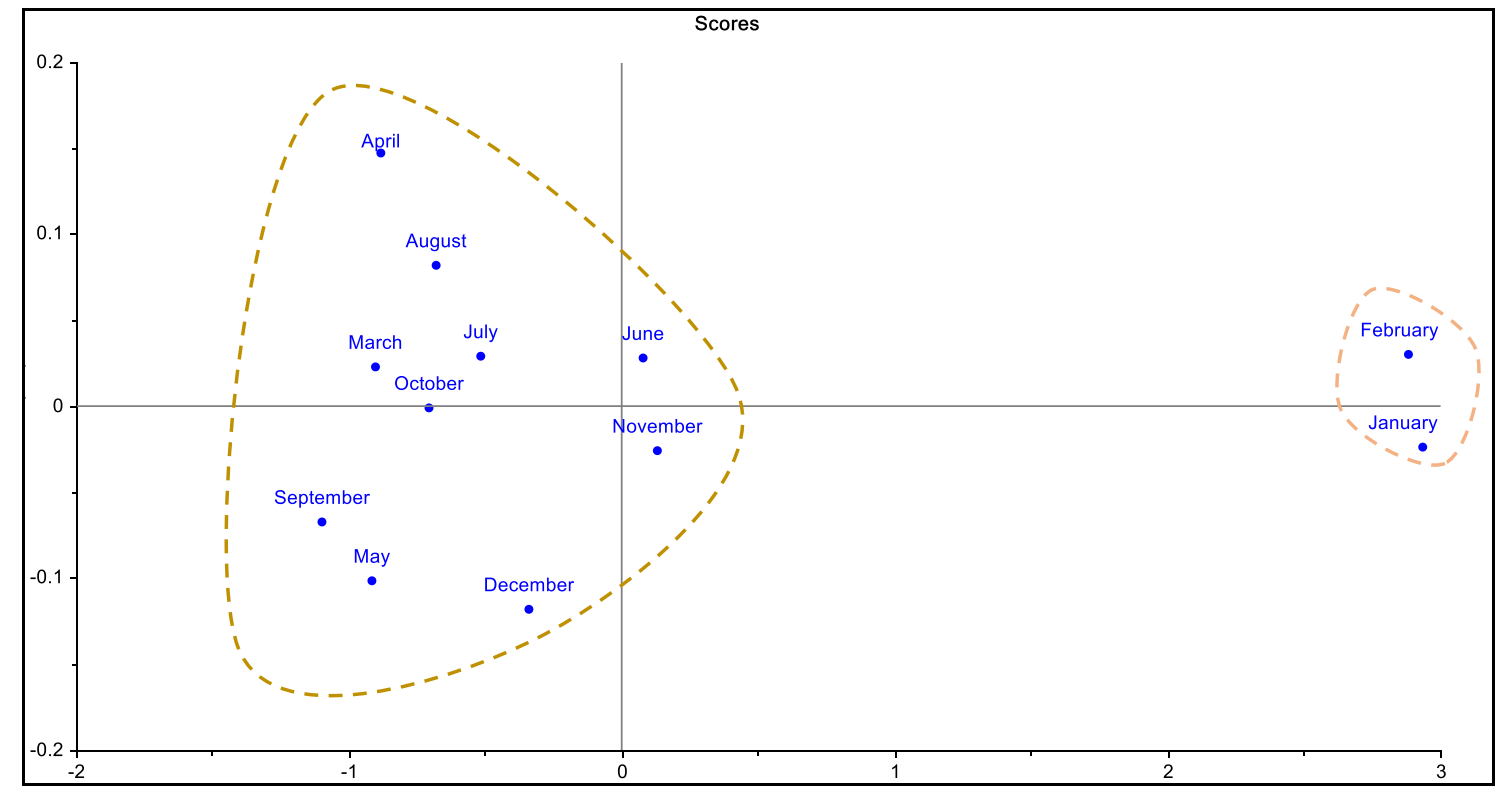

Figure 2. Principal component analysis (PCA) scores plot of the Relax batches samples (collected during months of 2017) measured by ET.

The data of ET was also analyzed using HCA. The dendrogram of HCA classifications is shown in Figure 3. It can be noticed that HCA has mainly two groups (i.e. A and B). The first group (i.e. A) represents the product in January-February and the second group (i.e. B) is for batches of March-December months. It can also be noticed that there are subgroups (i.e. B1 
and B2). However, the distance between them is small, and this indicates that there is homogeneity in the production and the shelf life has not much effect on the product quality.

The classification (two main groups in HCA) is expected to be due to the same reason that PCA managed to classify the two groups, i.e. changing in the product composition, which is due to stop adding mahaleb cherry to the mixture ingredients from the end of February month.

From data analysis viewpoint, it can be that both MVDA techniques managed to get the same interoperation of results.

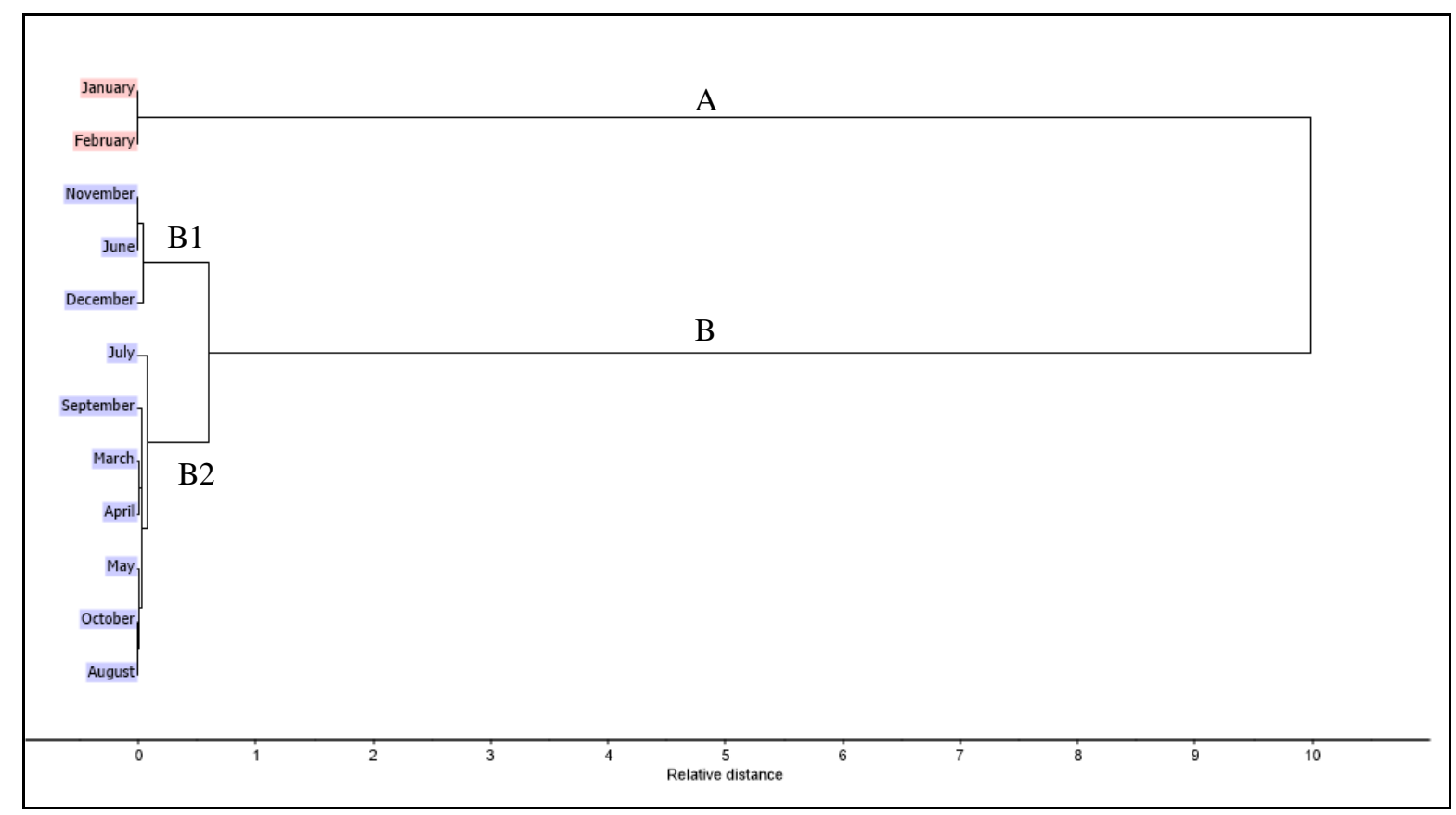

Figure 3. Dendrogram showing clustering of Relax herbal medicinal samples measure by ET. Two groups (A and B) are presented. The group in which mahaleb cherry was added to ingredients (A: in pink colour), and the group in which mahaleb cherry was not added to ingredients (B: in violet colour). Subgroups of B (i.e. B1 and B2) have little differences between them, which indicates that the product is homogeneous and there is a little effect of shelf life.

This study produced results which corroborate the findings of a great deal of the previous work in using ET for herbal products, tea [9,29,31], biotechnology and pharmaceutical applications [14,19,20,23,27]. Moreover, the present study extends the knowledge of using ET for different applications.

\section{Conclusion}

In this investigation, samples of local herbal medicinal plant product called Relax were measured by electronic tongue (ET) to check its quality and benchmarking during one year (i.e. 2017) of production. From the results obtained, it can be concluded that:

1) ET was able to follow the herbal medicinal plant storage time (shelf life). 
2) The ET signals indicated that there was little effect of the storage time on the homogeneity of the product, and this indicates the proper storage in the manufacturing plant.

3) ET was also successful in predicting the changing in the ingredients of the product (i.e. benchmarking), even though the change was in one compound that usually added in a small amount.

4) Both PCA and HCA techniques were able to classify the samples according to the internal characteristics (composition) of the herbal medicinal plant products.

ET showed high efficiency in quality control of Relax product. Further studies are needed to develop innovative analytical methods using ET with other herbal medicinal plants/products and other biotechnology applications.

\section{Acknowledgement}

The authors would like to thank Bajjora Company (Tulkarm, Palestine) for their cooperation during the project. This work has been partially financed by Dutch Nuffic through NICHEPAA-233 project. We would also like to thank Palestine Technical University-Kadoorie (PTUK) for supporting this research.

\section{References}

[1] van Wyk BE, Wink M., 2015. Phytomedicines, herbal drugs, and poisons. University of Chicago Press.

[2] Silva M. A., Higino J. S., Pereira J. V., Siqueira-Junior J. P. and Pereira M. S., 2008. Antibiotic activity of the extract of Punica granatum Linn. over bovine strains of Staphylococcus aureus. Revista Brasileira de Farmacognosia. 18, 209-212.

[3] Zampini I. C., Cuello S., Alberto M. R., Ordonez R. M., Dalmeida R., Solorzano E. and Isla M. I., 2009. Antimicrobial activity of selected plant species from -the Argentine Puna- against sensitive and multi-resistant bacteria. Journal of Ethnopharmacology. 124, 499-505.

[4] Asolini F. C., Tedesco A. M., Carpes S. T., Ferraz C. and Alencar S. d., 2006. Antioxidant and antibacterial activities of phenolic compounds from extracts of plants used as tea. Brazilian Journal of Food Technology. 9, 209-215.

[5] Proestos C., Lytoudi K., Mavromelanidou O. K., Zoumpoulakis P. and Sinanoglou V. J., 2013. Antioxidant capacity of selected plant extracts and their essential oils. Antioxidants. 2, 11-22.

[6] Sabbobeh R., Hejaz H., Jahajha A., Al-Akhras S., Al-Jaas H. and Abu-Lafi S., 2016. Antioxidant an Antimicrobial Activities of the Leaf Extract of Salvia palaestina. Journal of Applied Pharmaceutical Science. 6, 076-082.

[7] Gavali J. B., Pradhan N. and Waghmare N., 2016. Current Trends in Analytical Methods of Medicinal Plant Drugs. Jyoti Brijal Gavali et al; Current Trends in Analytical Methods of Medicinal Plant Drugs. 1, 101-107. 
[8] Ramautar R., Somsen G. W. and de Jong G. J., 2013. CE-MS for metabolomics: Developments and applications in the period 2010-2012. Electrophoresis. 34, 8698.

[9] Papieva I. S., Kirsanov D. O., Legin A. V., Kartsova L. A., Alekseeva A. V., Vlasov Y. G., Bhattacharyya N., Sarkar S. and Bandyopadkhyay R., 2011. Analysis of tea samples with a multisensor system and capillary electrophoresis. Russian Journal of Applied Chemistry. 84, 964-971.

[10] Yaroshenko I. S., Kirsanov D. O., Wang P., Ha D., Wan H., He J., Vlasov Y. G. and Legin A. V., 2015. Determination of the toxicity of herb preparations of the traditional Chinese medicine with a multisensor system. Russian Journal of Applied Chemistry. 88, 72-81.

[11] Major N., Markoviç K., Krpan M., Saric G., Hruskar M. and Vahcic N., 2011. Rapid honey characterization and botanical classification by an electronic tongue. Talanta. $85,569-574$.

[12] Kalit M. T., Markovic K., Kalit S., Vahcic N. and Havranek J., 2014. Application of electronic nose and electronic tongue in the dairy industry. Mljekarstvo. 64, 228244.

[13] Men H., Ning K. and Chen D., 2013. Data Fusion of Electronic Nose and Electronic Tongue for Discrimination of Chinese Liquors. Sensors \& Transducers. 157, 57-67.

[14] Ramamoorthy H. V., Mohamed S. N. and Devi D. S., 2014. E-Nose and E-Tongue: Applications and Advances in Sensor Technology. Journal of NanoScience and NanoTechnology. 2, 370-376.

[15] Wang P., Liu Q., Wu C. and Hsia K. J., 2015. Electronic Nose and Electronic Tongue. In: Wang, P., Liu, Q., Wu, C., Hsia, K.J. Eds., Bioinspired Smell and Taste Sensors, Springer. pp 19-44.

[16] Abu-Khalaf N., Iversen L. J. J., 2007. Calibration of a sensor array (an electronic tongue) for identification and quantification of odorants from livestock buildings. Sensors. 7, 103-128.

[17] Abu-Khalaf N., Iversen L. J. J., 2007. Classification of mixtures of odorants from livestock buildings by a sensor array (an electronic tongue). Sensors. 7, 129-143.

[18] Abu Rumaila B., 2019. Studying the Possibility of Indirect Metabolite Microorganisms' Classification Using Electronic Tongue and Multivariate Data Analysis. In: Master Thesis in Agricultural Biotechnology, Palestine Technical University - Kadoorie (PTUK), 159 pp.

[19] Abu-Khalaf N., Zaid A. N., Jaradat N., Alkilany A. M., Abulateefeh S. R., Al Ramahi R. and Ghanem M., 2019. Identification of substandard drug products using electronic tongue: cefdinir suspension as a pilot example. Drug Design, Development and Therapy. 13, 3249-3258.

[20] Abu-Khalaf N., Zaid N. A., Jaradat N., AlKilany A., Abu Rumaila B., Al Ramahi R., Shweiki S., Nidal S. and Surakhi N., 2018. The Taste of Commercially Available Clarithromycin Oral Pharmaceutical Suspensions in the Palestinian Market: Electronic Tongue and In Vivo Evaluation. Sensors. 18, 454-464.

[21] Al Ramahi R., Zaid A. N. and Abu-Khalaf N., 2019. Evaluating the potential use of electronic tongue in early identification and diagnosis of bacterial infections. Infection and Drug Resistance. 12, 2445-2451. 
[22] Soderstrom C., Winquist F. and Krantz-Rulcker C., 2003. Recognition of six microbial species with an electronic tongue. Sensors and Actuators B: Chemical. 89, 248-255.

[23] Voitechovic E., Korepanov A., Kirsanov D. and Legin A., 2018. Quantification of immobilized protein in pharmaceutical production by bio-assisted potentiometric multisensor system. Journal of Pharmaceutical and Biomedical Analysis. 150, 6771.

[24] Soderstrom C., Rudnitskaya A., Legin A. and Krantz-Rulcker C., 2005. Differentiation of four aspergillus species and one Zygosaccharomyces with two electronic tongues based on different measurement techniques. Journal of Biotechnology. 119, 300308.

[25] Soderstrom C., Boren H. and Krantz-Rulcker C., 2005. Use of an electronic tongue and HPLC with electrochemical detection to differentiate molds in culture media. International Journal of Food Microbiology. 97, 247-257.

[26] Soderstrom C., 2003. Measuring microbial acidity with electronic tongue. In: Institute of Technology, Linkoping University. Ph.D. thesis.

[27] Qneibi M., Jaradat N., Zaid A. N., Abu-Khalaf N., Natsheh A. R. and Hussen F., 2018. Evaluation of taste, total phenols and antioxidant for fresh, roasted, shade dried and boiled leaves of edible Arum palaestinum Bioss. Marmara Pharmaceutical Journal. 22, 52-58.

[28] Soderstrom C., Boren H., Winquist F. and Krantz-Rulcker C., 2003. Use of an electronic tongue to analyze mold growth in liquid media. International Journal of Food Microbiology. 83, 253-261.

[29] Saha P., Ghorai S., Tudu B., Bandyopadkhyay R. and Bhattacharyya N., 2020. Electronic Tongue for Tea Quality Assessment: Advanced Signal Processing Techniques for Estimation of Thearubigin and Theaflavin. In: Bhattacharya S Ed, Advancements in Instrumentation and Control in Applied System Applications, IGI Global. pp 108128.

[30] Taha H., 2019. Testing herbal medicines plants mixtures using a taste sensor "an electronic tongue" and multivariate data analysis. In: Master Thesis in Agricultural Biotechnology, Palestine Technical University - Kadoorie (PTUK), 117 pp.

[31] Adnan M., Ahmad A., Ahmed A., Khalid N., Hayat I. and Ahmed I., 2013. Chemical composition and sensory evaluation of tea (Camellia sinensis) commercialized in Pakistan. Pak J Bot. 45, 901-907.

[32] Alpha MOS., 2009. Astree II manual: Taste sensor. Toulouse, France, Alpha MOS. In: https://www.alpha-mos.com/astree-taste-analysis.

[33] Abu-Khalaf N., Khayat S. and Natsheh B., 2013. Multivariate data analysis to identify the groundwater pollution sources in Tulkarm area / Palestine. Science and Technology. 3, 99-104. 